\title{
Efeito da adição do 2,4-D ao glyphosate para o controle de espécies de plantas daninhas de difícil controle ${ }^{1}$
}

\author{
Effect of 2.4-D addition to glyphosate for difficult control weeds species
}

\author{
Hudson Kagueyama Takano ${ }^{2}$; Rubem Silvério de Oliveira Jr. ${ }^{3}$; Jamil Constantin ${ }^{3}$; Denis \\ Fernando Biffe ${ }^{3}$; Luiz Henrique Morais Franchini ${ }^{4}$; Guilherme Braga Pereira Braz ${ }^{4}$; Fabiano \\ Aparecido Rios ${ }^{4}$; Eliezer Antonio Gheno ${ }^{4}$; Alexandre Gemelli ${ }^{4}$
}

Resumo - O 2,4-D tem sido um dos herbicidas mais utilizados em associação com o glyphosate, principalmente nas aplicações de dessecação em pré-plantio. O objetivo deste trabalho foi avaliar o efeito da adição do 2,4-D ao glyphosate visando o controle de espécies de plantas daninhas consideradas de difícil controle (Commelina benghalensis, Richardia brasiliensis, Euphorbia heterophylla, Spermacoce latifolia, Ipomoea grandifolia e Conyza spp.). O delineamento experimental foi o inteiramente casualizado, e os tratamentos foram arranjados em esquema fatorial $3 \times 4$, com quatro repetições. O primeiro fator foi constituído por estádios de desenvolvimento das plantas daninhas (2-4, 4-6 e >10 folhas) e o segundo fator por tratamentos com glyphosate (720 g i.a. ha $\left.{ }^{-1}\right), 2,4-\mathrm{D}\left(670 \mathrm{~g}\right.$ i.a. ha $\left.{ }^{-1}\right)$ glyphosate+2,4-D $\left(720+670 \mathrm{~g}\right.$ i.a. ha $\left.{ }^{-1}\right)$ e um tratamento sem herbicida (testemunha). Para a buva em casa-de-vegetação, foram realizados mais três tratamentos herbicidas adicionais variando as doses de glyphosate e 2,4-D. A adição de 2,4-D ao glyphosate foi determinante para acelerar e melhorar o controle de plantas daninhas de difícil controle estudadas.

Palavras-chaves: sinergismo, velocidade de controle, mistura em tanque

\begin{abstract}
The 2,4-D has been one of the most widely used herbicide in combination with glyphosate, especially in applications desiccation pre-planting. The objective of this study was to evaluate the effect of adding 2,4-D to glyphosate for the control of weed species considered difficult to control (Commelina benghalensis, Richardia brasiliensis, Euphorbia heterophylla, Spermacoce latifolia, Ipomoea grandifolia e Conyza spp.). The experimental design was completely randomized, and treatments were arranged in a $3 \times 4$ factorial design with four replications. The first factor was composed of stages of development of weeds (2-4, 4-6 e >10 leaves) and the second factor by treatment with glyphosate $\left(720 \mathrm{~g}\right.$ i.a. ha $\left.{ }^{-1}\right), 2,4-\mathrm{D}\left(670 \mathrm{~g}\right.$ i.a. ha $\left.{ }^{-1}\right)$ glyphosate+2,4-D $\left(720+670 \mathrm{~g}\right.$ i.a. ha $\left.{ }^{-1}\right)$ and a treatment without herbicide (control). For Conyza spp. in green house, were performed three additional herbicide treatments varying doses of glyphosate and 2,4-D. The addition of 2,4-D to glyphosate is crucial to accelerate and improve weed control considered unwieldy as those studied in this work.
\end{abstract}

Keywords: synergism, speed control, tank mixtures

\footnotetext{
${ }^{1}$ Recebido para publicação em 09/02/2013 e aceito em 10/04/2013.

2 Acadêmico do curso de Agronomia da Universidade Estadual de Maringá (UEM/NAPD), Av. Colombo 5790, 87020-900, Maringá, PR. Email: <hudsontakano@gmail.com>. (Autor para correspondência).

${ }^{3}$ Professores do Departamento de Agronomia da Universidade Estadual de Maringá (UEM/NAPD).

${ }^{4}$ Doutorandos do Programa de Pós-Graduação em Agronomia da Universidade Estadual de Maringá (UEM/NAPD).
} 


\section{Introdução}

Com o advento das culturas geneticamente modificadas para a tolerância a herbicidas, o glyphosate ganhou amplo espaço no manejo de plantas daninhas dentro do ciclo das culturas. Porém, espécies de difícil controle como erva-quente (Spermacoce latifolia), trapoeraba (Commelina benghalensis), poaia (Richardia brasiliensis), leiteiro (Euphorbia heterophylla), corda-de-viola (Ipomea grandifolia) e buva (Conyza spp.) vêm sendo selecionadas devido a aplicações sucessivas de glyphosate em áreas agrícolas.

Correia et al. (2008) verificaram que mesmo utilizando doses elevadas de glyphosate $\left(1200\right.$ g e.a. ha $\left.{ }^{-1}\right)$ o controle exercido por este herbicida em plantas de Commelina benghalensis (4 a 6 folhas) foi baixo $(42,5 \%)$. Carvalho et al. (2002) também relataram que a aplicação de glyphosate a 760 e a 940 g e.a. ha 1 proporcionou controle insatisfatório de Euphorbia heterophylla com 8 a 10 folhas aos 30 DAA, não ultrapassando 72,5 e 77,0\% de controle, respectivamente. Segundo Ramires et al. (2010), a aplicação de glyphosate isolado $\left(960 \mathrm{~g}\right.$ e.a. ha $\left.{ }^{-1}\right)$ não exerceu controle satisfatório de plantas de Ipomoea grandifolia com 4-6 folhas, sendo que aos 28 DAA a porcentagem de controle foi de $76,2 \%$.

Em relação à Spermacoce latifolia, alguns autores têm relatado evidencias da tolerância dessa espécie ao glyphosate mesmo em estádios de desenvolvimento mais precoces (Ramires et al. 2011; Monquero et al. 2005).

Com relação à buva (Conyza spp.) observa-se que o controle não é satisfatório $(54,8 \%)$ mesmo em aplicações sequenciais de glyphosate à $720 \mathrm{~g}$ e.a. ha ${ }^{-1}$ (Yamauti et al., 2010). Além disso, autores como Vargas et al (2007) e Santos et al. (2012) comprovaram a resistência desta planta daninha ao glyphosate.

As falhas no controle de determinadas espécies de plantas daninhas pelo uso do glyphosate têm levado agricultores a utilizar outros herbicidas mesmo em lavouras com a tecnologia RR. O 2,4-D tem sido um dos herbicidas mais utilizados em associação com o glyphosate, principalmente nas aplicações de dessecação pré-plantio. Apesar disso, não há resultados expressos de forma clara sobre os efeitos da adição do 2,4-D ao glyphosate no controle de plantas daninhas consideradas de difícil controle.

Dentro deste contexto, o objetivo do trabalho foi avaliar o efeito da adição do 2,4-D ao glyphosate no controle de espécies de plantas daninhas consideradas de difícil controle.

\section{Material e Métodos}

Foram realizados experimentos em casa de vegetação no Centro de Treinamento em Irrigação (CTI/UEM) localizado no município de Maringá-PR, e a campo no Sítio São Pedro, em Pulinópolis, distrito de Mandaguaçu-PR a $23^{\circ} 14^{\prime} 31.81^{\prime \prime}$ de latitude Sul e 52 $02^{\prime} 33.05^{\prime \prime}$ de longitude Oeste, à 462 metros de altitude. $\mathrm{O}$ período de condução dos ensaios foi de 16/08/2012 a 08/12/2012.

Em casa de vegetação foram realizados seis experimentos, sendo cada experimento constituído por uma espécie de planta daninha, cujas as unidades experimentais foram compostas por vasos com capacidade para 3 $\mathrm{dm}^{3}$, os quais foram preenchidos com solo com as seguintes características: $\mathrm{pH}$ em água de 5,$90 ; 3,68 \mathrm{cmol}_{\mathrm{c}}$ de $\mathrm{H}^{+}+\mathrm{Al}^{+3} \mathrm{dm}^{-3}$ de solo; $3,17 \mathrm{cmol}_{\mathrm{c}} \mathrm{dm}^{-3}$ de $\mathrm{Ca}^{+2} ; 0,67 \mathrm{cmol}_{\mathrm{c}} \mathrm{dm}^{-3} \mathrm{de}$ $\mathrm{Mg}^{+2} ; 0,61 \mathrm{cmol}_{\mathrm{c}} \mathrm{dm}^{-3}$ de $\mathrm{K}^{+} ; 47,60 \mathrm{mg} \mathrm{dm}^{-3} \mathrm{de}$ P; $11,89 \mathrm{~g} \mathrm{dm}^{-3}$ de C; $640 \mathrm{~g} \mathrm{~kg}^{-1}$ de areia grossa; $50 \mathrm{~g} \mathrm{~kg}^{-1}$ de areia fina; $20 \mathrm{~g} \mathrm{~kg}^{-1} \mathrm{de}$ silte e $290 \mathrm{~g} \mathrm{~kg}^{-1}$ de argila.

As espécies avaliadas foram trapoeraba (Commelina benghalensis), poaia (Richardia brasiliensis), leiteiro (Euphorbia heterophylla), erva-quente (Spermacoce latifolia), corda-deviola (Ipomoea grandifolia) e buva (Conyza spp.). O delineamento experimental foi $\mathrm{o}$ inteiramente casualizado. Os tratamentos foram arranjados em esquema fatorial $3 \times 4$, com quatro repetições, onde o primeiro fator representou três estádios de desenvolvimento 
das espécies de plantas daninhas (Tabela 1) e o segundo fator por quatro tratamentos herbicidas (Tabela 2). Para a buva em casa-devegetação, foram realizados mais três tratamentos herbicidas adicionais: glyphosate+2,4-D (720+670), glyphosate+2,4-
D (720+1005), glyphosate+2,4-D (900+670) e glyphosate+2,4-D $(900+1005)$. Vale ressaltar que em casa-de-vegetação foi utilizado um biótipo de buva proveniente de uma população suscetível ao glyphosate, ao passo no campo o biótipo era considerado resistente.

Tabela 1. Estádios de desenvolvimento das plantas daninhas nos quais foram realizadas as aplicações de herbicidas. Maringá-PR/2012.

\begin{tabular}{|c|c|c|c|}
\hline \multirow{2}{*}{ Espécie } & \multicolumn{3}{|c|}{ Estádios de desenvolvimento } \\
\hline & E1 & E2 & $\mathbf{E 3}$ \\
\hline Trapoeraba & 2-4 folhas & 4-6 folhas & $>10$ folhas \\
\hline Poaia & $2-4$ folhas & 4-6 folhas & $>10$ folhas \\
\hline Erva-quente & 2-4 folhas & 4-6 folhas & $>10$ folhas \\
\hline Corda de viola & $2-4$ folhas & 4-6 folhas & $>10$ folhas \\
\hline Leiteiro & 2-4 folhas & 4-6 folhas & $>10$ folhas \\
\hline Buva & $<6 \mathrm{~cm}$ & $6-15 \mathrm{~cm}$ & $>15 \mathrm{~cm}$ \\
\hline
\end{tabular}

Tabela 2. Tratamentos herbicidas utilizados nos seis experimentos. Maringá-PR/2012.

\begin{tabular}{|c|c|c|c|}
\hline \multirow{2}{*}{ Tratamentos } & \multirow{2}{*}{ Produto comercial } & \multicolumn{2}{|c|}{ Doses } \\
\hline & & $\left(\right.$ g e.a. ha $\left.{ }^{-1}\right)$ & (L p.c. ha $\left.{ }^{-1}\right)$ \\
\hline 1. Testemunha & - & - & - \\
\hline 2. Glyphosate & Roundup Original & 720 & 2,0 \\
\hline 3. $2,4-\mathrm{D}$ & DMA 806 BR & 670 & 1,0 \\
\hline 4. Glyphosate + 2,4-D & Roundup Original + DMA 806 BR & $720+670$ & $2,0+1,0$ \\
\hline
\end{tabular}

Para cada espécie foram distribuídas aproximadamente 30 sementes por vaso, a uma profundidade de 1 a $3 \mathrm{~cm}$ em relação ao nível do solo. Após a emergência das plantas daninhas, efetuou-se o desbaste nas unidades experimentais deixando de duas a quatro plantas por vaso. Até a avaliação final de controle, as unidades experimentais foram submetidas a irrigação diária, sendo mantidas próximos à capacidade de campo.

No experimento a campo, as unidades experimentais foram compostas por parcelas de $4 \mathrm{x} 4 \mathrm{~m}\left(16 \mathrm{~m}^{2}\right)$, nas quais o solo apresentou valores de $\mathrm{pH}$ em água de 6,$10 ; 3,56 \mathrm{cmol}_{\mathrm{c}}$ de $\mathrm{H}^{+}+\mathrm{Al}^{+3} \mathrm{dm}^{-3}$ de solo; 4,02 cmol $_{\mathrm{c}} \mathrm{dm}^{-3} \mathrm{de}$ $\mathrm{Ca}^{+2} ; 0,71 \mathrm{cmol}_{\mathrm{c}} \mathrm{dm}^{-3}$ de $\mathrm{Mg}^{+2} ; 0,63 \mathrm{cmol}_{\mathrm{c}} \mathrm{dm}^{-3}$ de $\mathrm{K}^{+} ; 43,80 \mathrm{mg} \mathrm{dm}^{-3}$ de P; $13,99 \mathrm{~g} \mathrm{dm}^{-3}$ de C; $140 \mathrm{~g} \mathrm{~kg}^{-1}$ de areia grossa; $280 \mathrm{~g} \mathrm{~kg}^{-1}$ de areia fina; $20 \mathrm{~g} \mathrm{~kg}^{-1}$ de silte e $540 \mathrm{~g} \mathrm{~kg}^{-1}$ de argila. $\mathrm{O}$ delineamento experimental utilizado foi o de blocos casualizados com quatro repetições, e as espécies presentes na área foram Conyza spp.
(E1, E2 e E3) e Commelina benghalensis (E3), em densidades de 48 e de 3 plantas $\mathrm{m}^{-2}$, respectivamente.

Para as aplicações dos tratamentos em ambos os locais foi utilizado um pulverizador costal de pressão constante à base de $\mathrm{CO}_{2}$, equipado com barra munida de três pontas tipo jato leque XR-110.02, espaçadas a $50 \mathrm{~cm}$ entre si, sob pressão de $2 \mathrm{kgf} \mathrm{cm}^{-2}$. Estas condições de aplicação proporcionaram o equivalente a $200 \mathrm{~L} \mathrm{ha}^{-1}$ de calda. No momento da aplicação, as condições climáticas foram constituídas por: Temperatura de $27,0^{\circ} \mathrm{C}$ e $28,5^{\circ} \mathrm{C}$; umidade relativa 65 e $60 \%$ e velocidade dos ventos de 2,0 e $1,1 \mathrm{~km} \mathrm{~h}^{-1}$ na casa-de-vegetação e no campo, respectivamente.

Foram realizadas avaliações de controle aos 7, 14, 21 e 28 dias após a aplicação (DAA), usando escala em que $0 \%$ representa ausência de efeito do herbicida sobre as plantas, e 100\% representa morte das plantas. Em casa-devegetação as plantas daninhas trapoeraba, poaia 
e buva no estádio E3 foram avaliadas até 56, 42 e 42 DAA, respectivamente. No campo, as avaliações foram realizadas até 42 DAA.

Os dados da última avaliação de controle de cada espécie foram submetidos à análise de variância pelo teste F. Efeitos significativos foram submetidos à comparação das médias pelo teste Tukey a $5 \%$ de probabilidade, utilizando-se o programa estatístico SISVAR. Os dados de controle ao longo das avaliações foram representados por meio de figuras, visando avaliar comparativamente a velocidade de controle dos tratamentos.

Os dados de controle foram submetidos ainda à análise pelo modelo proposto por Colby (1967), utilizando a equação descrita por Monquero et al. (2001), para a avaliação dos efeitos sinergísticos e antagonísticos entre as associações herbicidas testadas.

\section{Resultados e Discussão}

$\mathrm{Na}$ última avaliação realizada em casade-vegetação (28 DAA), observou-se que para C. benghalensis, quanto maior o estádio de desenvolvimento, mais difícil é o seu controle (Tabela 3). Aplicações realizadas no estádio E1 não apresentaram diferenças significativas entre os tratamentos com herbicidas, sendo todos eles eficazes no controle da trapoeraba. Já nos estádios E2 e E3, a associação entre o glyphosate e 2,4-D exerceu controle final superior aos herbicidas aplicados de forma isolada. Observa-se que para plantas com mais de 10 folhas, nem mesmo a associação entre os dois herbicidas foi capaz de apresentar níveis de controle satisfatórios aos 28 DAA, sendo necessário maior período para se visualizar os resultados finais (Tabela 4). No entanto, mesmo neste caso a associação entre os dois herbicidas apresentou controle final superior aos herbicidas aplicados isoladamente.

O controle final de $C$. benghalensis foi semelhante nas avaliações de campo (Tabela 4) e casa-de-vegetação. Constatou-se que a associação entre o glyphosate e o 2,4-D foi superior ao 2,4-D isolado, que por sua vez exerceu controle melhor que o glyphosate também aplicado de forma isolada. Nestas avaliações, somente a associação entre os herbicidas foi capaz de proporcionar controle aceitável de trapoeraba, evidenciando a eficácia desta mistura em espécies que o glyphosate tem dificuldade em controlar quando aplicado isoladamente. Estes resultados estão de acordo com Ramos \& Durigan (1996), os quais relatam que a mistura de glyphosate $+2,4-\mathrm{D}$ apresentou controle superior da trapoeraba (Commelina virginica L.), em relação aos produtos aplicados isoladamente. Os resultados de campo e de casa-de-vegetação foram semelhantes, mas o controle final atingido em plantas bem desenvolvidas foi maior em casade-vegetação, provavelmente em função do menor nível de estresse fisiológico que as plantas sofreram em relação as desenvolvidas em condição de campo.

Tomando como base o controle da espécie proporcionado por cada herbicida aplicado isoladamente, o método de Colby estima um valor de controle teórico que deveria ser alcançado pela mistura entre dois herbicidas. Na Tabela 3 observa-se que o controle estimado da mistura glyphosate+2,4-D para as plantas do estádio E1 seria de 99,8\%, e como o controle obtido foi maior $(100,0 \%)$, conclui-se que a mistura apresentou efeito sinergístico. Analisando as interações para a mistura de glyphosate com o 2,4-D pelo modelo de Colby (1967) nas Tabelas 3 e 4, observaram-se efeitos sinergísticos proporcionados por esta mistura nas avaliações realizadas em campo e casa-de-vegetação (28, 42 e 56 DAA).

Com relação à velocidade de controle (Figura 1), observou-se que para todos os estádios de desenvolvimento (E1, E2 e E3) o tratamento com a associação de glyphosate e 2,4-D proporcionou controle mais rápido quando comparado a aplicação dos herbicidas de forma isolada. Quando os tratamentos foram aplicados em estádio precoces (E1), a diferença 
de velocidade de controle foi pequena. Porém, em aplicações mais tardias (E2 e E3), além do controle inicial ser evidentemente superior com a mistura, a diferença entre a velocidade de controle da mistura em relação aos produtos isolados tende a ser mais evidente.

Tabela 3. Controle de Commelina benghalensis proporcionado pelos tratamentos herbicidas aos 28 DAA (experimento em casa-de-vegetação). Maringá (PR), 2012.

\begin{tabular}{|c|c|c|c|}
\hline \multirow{2}{*}{ Herbicidas e doses (g e.a. ha $^{-1}$ ) } & \multicolumn{3}{|c|}{ Estádio da planta daninha no momento das aplicações* } \\
\hline & 2-4 folhas (E1) & 4-6 folhas (E2) & $>10$ folhas $(\mathrm{E3})$ \\
\hline 1. Testemunha sem herbicida & $0,00 \quad \mathrm{bA}$ & $0,00 \quad \mathrm{cA}$ & $0,00 \quad \mathrm{cA}$ \\
\hline 2. Glyphosate (720) & $98,70 \quad \mathrm{aA}$ & $82,00 \quad b B$ & 6,20 \\
\hline 3. $2,4-\mathrm{D}(670)$ & 97,50 & 74,20 & 27,50 \\
\hline 4. Glyphosate+2,4-D (720+670) & $100,00 \quad \mathrm{aA}$ & 100,00 & 62,50 \\
\hline Estimativa de Colby & 99,87 & 95,34 & 32,12 \\
\hline $\begin{array}{l}\text { DMS linha }=10,07 \\
\text { DMS coluna }=9,41 \\
C V \%=11,03\end{array}$ & & & \\
\hline
\end{tabular}

Tabela 4. Comparativo dos níveis de eficácia de controle de Commelina benghalensis (apenas para plantas que receberam aplicação no estádio E3) proporcionados pelos tratamentos herbicidas aplicados a campo e em casa-de-vegetação. Maringá (PR), 2012.

\begin{tabular}{|c|c|c|c|c|}
\hline \multirow{2}{*}{ Herbicidas e doses (g e.a. ha $^{-1}$ ) } & \multicolumn{4}{|c|}{$\%$ Controle } \\
\hline & 42 DAA (campo) & & 56 DAA (casa-de-ve & getação) \\
\hline 1. Testemunha sem herbicida & 0,0 & $\mathrm{~d}$ & 0,00 & $\mathrm{D}$ \\
\hline 2. Glyphosate (720) & 37,50 & $\mathrm{c}$ & 46,20 & $\mathrm{C}$ \\
\hline 3. $2,4-\mathrm{D}(670)$ & 50,00 & $\mathrm{~b}$ & 57,50 & $\mathrm{~B}$ \\
\hline 4. Glyphosate+2,4-D $(720+670)$ & 85,00 & $\mathrm{a}$ & 98,20 & A \\
\hline Estimativa de Colby & 68,75 & & 77,06 & \\
\hline $\mathrm{CV} \%$ & 6,77 & & 5,20 & \\
\hline
\end{tabular}

*Médias seguidas de mesma letra na coluna não diferem entre si pelo teste de Tukey a $5 \%$.

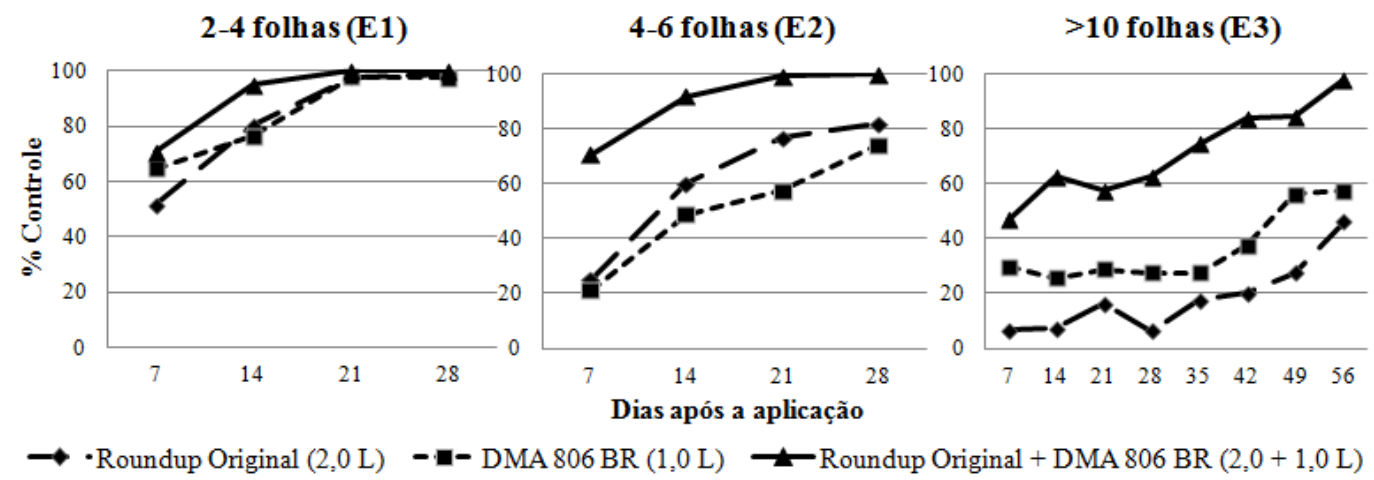

Figura 1. Controle de Commelina benghalensis após a aplicação de tratamentos herbicidas em três estádios de desenvolvimento ao longo do período de avaliação (Ensaio em casa-devegetação). Maringá (PR), 2012.

\section{Poaia (Richardia brasiliensis)}

O controle exercido pelos herbicidas sobre a espécie $R$. brasiliensis aos 28 DAA
(Tabela 5) foi semelhante nos menores estádios de desenvolvimento (E1 e E2). Já no estádio E3 observou-se que houve diferença entre a 
eficácia dos tratamentos testados, obedecendo a seguinte ordem crescente: 2,4-D, glyphosate, glyphosate+2,4-D. A aplicação de 2,4-D isolado em plantas de $R$. brasiliensis com mais de 10 folhas não proporcionou controle satisfatório aos 28 DAA. Nesta avaliação também foi constatado que quando os produtos foram aplicados isoladamente, houve maior dificuldade de controle à medida que se aumentou o estádio de desenvolvimento da planta daninha. Por outro lado, o controle proporcionado pelo glyphosate+2,4-D não diferiu entre os estádios de desenvolvimento de $R$. brasiliensis. Esses dados estão de acordo com Sharma \& Singh (2001), os quais obtiveram $100 \%$ de controle dessa espécie com 10 a $15 \mathrm{~cm}$, por meio da aplicação de
glyphosate+2,4-D. Foram observados efeitos sinergísticos entre a mistura de glyphosate e 2,4-D, uma vez que o controle exercido foi superior ao valor estimado pelo modelo de Colby (Tabela 5).

Com relação ao controle de Richardia brasiliensis (Tabela 6) em estádios de desenvolvimento avançados (E3) a aplicação de glyphosate e 2,4-D de forma isolada não proporcionou resultados satisfatórios $(\geq 80 \%)$. No entanto, a sua mistura provocou a morte total desta espécie aos 42 DAA. Comparando a porcentagem de controle proporcionada pela mistura em tanque de glyphosate+2,4-D e o valor estimado pelo modelo de Colby, observase que a mistura foi sinergística.

Tabela 5. Controle de Richardia brasiliensis proporcionado pelos tratamentos herbicidas aos 28 DAA (experimento em casa-de-vegetação). Maringá (PR), 2012.

\begin{tabular}{|c|c|c|c|}
\hline \multirow{2}{*}{ Herbicidas e doses (g e.a. ha $^{-1}$ ) } & \multicolumn{3}{|c|}{ Estádio* } \\
\hline & 2-4 folhas (E1) & 4-6 folhas (E2) & $>10$ folhas $(\mathrm{E3})$ \\
\hline 1. Testemunha sem herbicida & $0,00 \quad \mathrm{bA}$ & $0,00 \quad \mathrm{bA}$ & $0,00 \quad \mathrm{dA}$ \\
\hline 2. Glyphosate (720) & $100,00 \quad \mathrm{aA}$ & 97,50 & 82,50 \\
\hline 3. $2,4-\mathrm{D}(670)$ & $100,00 \quad \mathrm{aA}$ & 89,00 & 53,70 \\
\hline 4. Glyphosate+2,4-D $(720+670)$ & $100,00 \quad \mathrm{aA}$ & 99,70 & $100,00 \quad \mathrm{aA}$ \\
\hline Estimativa de Colby & 100,00 & 99,57 & 99,90 \\
\hline $\begin{array}{l}\text { DMS linha }=9,41 \\
\text { DMS coluna }=10,37 \\
C V \%=8,02\end{array}$ & & & \\
\hline
\end{tabular}

Tabela 6. Controle final (42 DAA) de Richardia brasiliensis (E3) proporcionado pelos tratamentos herbicidas (experimento em casa-de-vegetação). Maringá (PR), 2012.

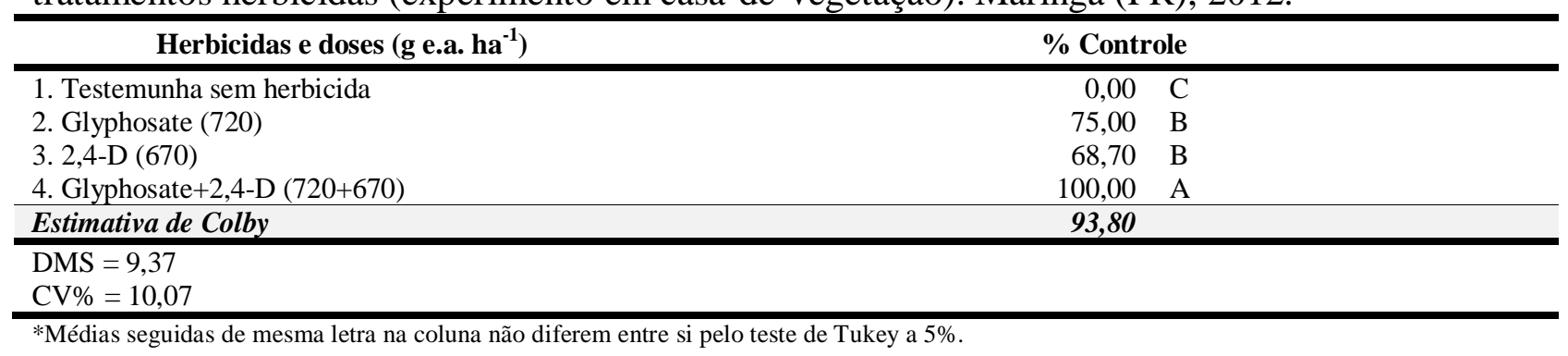

O tratamento com glyphosate isolado proporcionou o controle de $R$. brasiliensis mais rápido que a mistura em tanque de glyphosate+2,4-D e 2,4-D isolado em estádios menores (Figura 2). Segundo Monquero et al. (2001), existe uma suscetibilidade desta espécie, quando o glyphosate é aplicado em condições de pósemergência inicial da planta daninha. Por outro lado, em estádios mais tardios a mistura em tanque de glyphosate $+2,4-\mathrm{D}$ proporcionou 
controle muito mais rápido que os produtos aplicados de forma isolada.

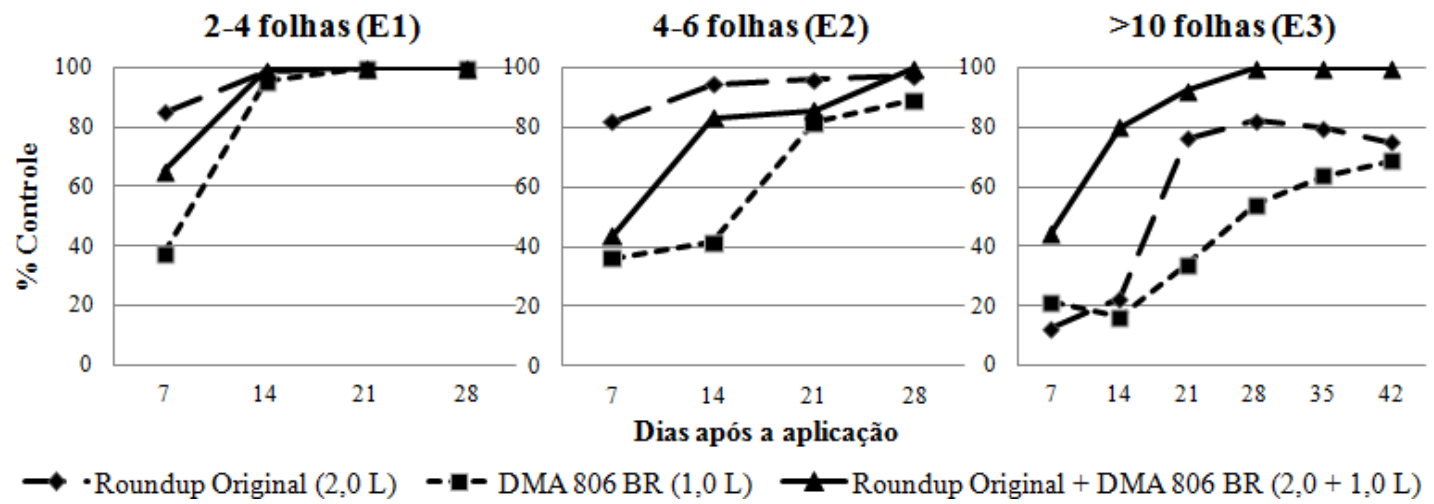

Figura 2. Controle de Richardia brasiliensis após a aplicação de tratamentos herbicidas em três estádios de desenvolvimento ao longo do período de avaliação (Ensaio em casa-de-vegetação). Maringá (PR), 2012.

\section{Leiteiro (Euphorbia heterophylla)}

Aos 28 DAA (Tabela 7) foi observado que para E1 e E2 não houve diferença no controle do leiteiro entre os tratamentos herbicidas. Porém, em E3 a aplicação de 2,4-D isolado proporcionou controle inferior, não sendo considerado como satisfatório. Além disso, o controle exercido por todos os herbicidas avaliados foi inferior quando aplicado em plantas de estádio mais avançado (E3). Carvalho et al. (2002) relataram que a aplicação de glyphosate+2,4-D (960+868) proporcionou controle semelhante à aplicação de glyphosate isolado (960), sendo as notas de eficiência foram 91,3 e $90 \%$ respectivamente, o que se assemelha ao observado no presente trabalho.

De acordo com o modelo proposto por Colby (1967), a associação entre o glyphosate e o 2,4-D foi aditiva para os estádios E1 e E2, enquanto que no estádio E3 a mistura foi sinergística (Tabela 7).

Nos estádios de desenvolvimento mais precoces (E1 e E2), a associação de glyphosate+2,4-D proporcionou maior velocidade inicial de controle em relação a aplicação dos produtos isolados (Figura 3). Já no estádio E3 a velocidade de controle foi semelhante com a aplicação de glyphosate isolado ou em associação com o 2,4-D.

Tabela 7. Controle de Euphorbia heterophylla proporcionado pelos tratamentos herbicidas aos 28 DAA (experimento em casa-de-vegetação). Maringá (PR), 2012.

\begin{tabular}{|c|c|c|c|}
\hline \multirow{2}{*}{ Herbicidas e doses (g e.a. ha ${ }^{-1}$ ) } & \multicolumn{3}{|c|}{ Estádio* } \\
\hline & 2-4 folhas (E1) & 4-6 folhas (E2) & $>10$ folhas $(\mathrm{E} 3)$ \\
\hline 1. Testemunha sem herbicida & $0,00 \quad \mathrm{bA}$ & $0,00 \quad \mathrm{bA}$ & $0,00 \quad \mathrm{cA}$ \\
\hline 2. Glyphosate (720) & $100,00 \quad \mathrm{aA}$ & 99,50 aA & 87,50 \\
\hline 3. $2,4-\mathrm{D}(670)$ & $100,00 \quad \mathrm{aA}$ & 99,20 & $47,00 \quad b B$ \\
\hline 4. Glyphosate+2,4-D (720+670) & $100,00 \quad \mathrm{aA}$ & 100,00 & $90,00 \quad \mathrm{aB}$ \\
\hline Estimativa de Colby & 100,00 & 100,00 & 87,43 \\
\hline $\begin{array}{l}\text { DMS linha }=4,44 \\
\text { DMS coluna }=4,89 \\
C V \%=3,81\end{array}$ & & & \\
\hline
\end{tabular}




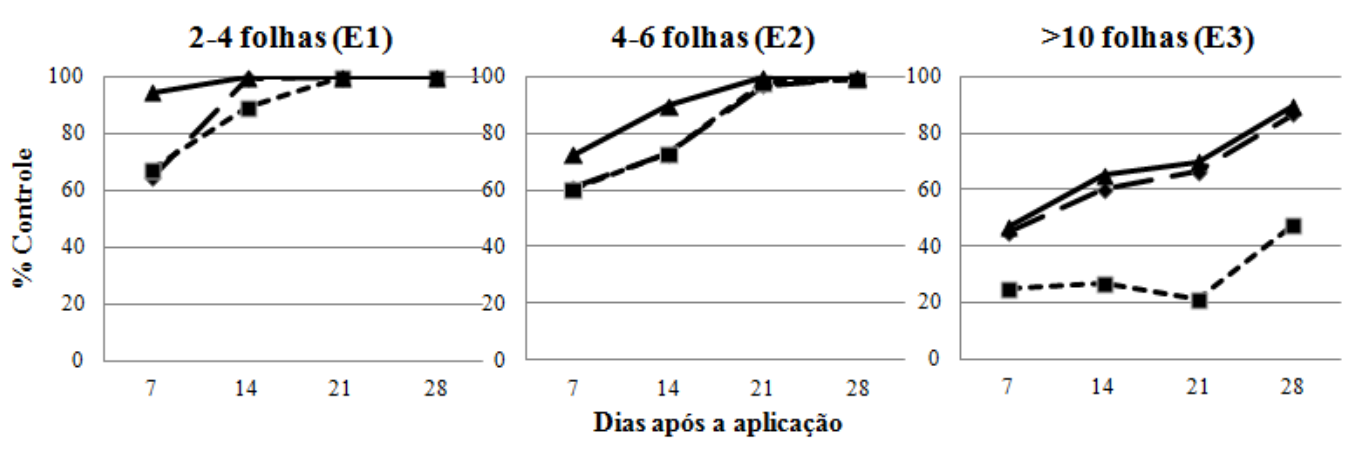

$\rightarrow$ Roundup Original (2,0 L) - -m- DMA 806 BR $(1,0$ L) $\rightarrow$ Roundup Original + DMA 806 BR $(2,0$ + 1,0 L)

Figura 3. Controle de Euphorbia heterophylla após a aplicação de tratamentos herbicidas em três estádios de desenvolvimento ao longo do período de avaliação (Ensaio em casa-de-vegetação). Maringá (PR), 2012.

\section{Erva-quente (Spermacoce latifolia)}

Aos 28 DAA, os tratamentos herbicidas não diferiram entre si quando aplicados nos estádios E1 e E2, sendo que todos provocaram a morte das plantas (Tabela 8). Já no estádio E3, a aplicação de 2,4-D isolado proporcionou controle inferior ao glyphosate isolado e a mistura glyphosate+2,4-D, não sendo considerado como satisfatório.

Foram observados efeitos aditivos entre a associação de glyphosate e 2,4-D em plantas com até 4-6 folhas (Tabela 8). Para o estádio E3 foi observado efeito sinergístico, pois o controle proporcionado pela mistura foi superior ao valor determinado pelo modelo de Colby (1967). Quanto maior o estádio no momento da aplicação, menor foi o controle exercido pelo 2,4-D.

A mistura em tanque de glyphosate+2,4-D proporcionou um controle inicial melhor do que os produtos aplicados isoladamente no estádio E1 (Figura 6). Por outro lado, apesar do controle aos 28 DAA ter sido semelhante para E2 e E3, a aplicação de glyphosate isolado controlou as plantas de $S$. latifolia mais rapidamente do que a associação de glyphosate+2,4-D. Ramires et al. (2011) também constataram excelentes níveis de controle exercidos pela aplicação de 960 g e.a $\mathrm{ha}^{-1}$ de glyphosate logo aos 7 DAA, em plantas de erva-quente constiuídas 4 a 6 folhas.

Tabela 8. Controle de Spermacoce latifolia proporcionado pelos tratamentos herbicidas aos 28 DAA (experimento em casa-de-vegetação) Maringá (PR), 2012.

\begin{tabular}{|c|c|c|c|}
\hline \multirow{2}{*}{ Herbicidas e doses (g e.a. ha ${ }^{-1}$ ) } & \multicolumn{3}{|c|}{ Estádio* } \\
\hline & 2-4 folhas (E1) & 4-6 folhas (E2) & $>10$ folhas $(\mathrm{E3})$ \\
\hline 1. Testemunha sem herbicida & $0,00 \quad \mathrm{bA}$ & $0,00 \quad \mathrm{bA}$ & $0,00 \quad \mathrm{cA}$ \\
\hline 2. Glyphosate (720) & $100,00 \quad \mathrm{aA}$ & 100,00 & 99,50 \\
\hline 3. $2,4-\mathrm{D}(670)$ & $100,00 \quad \mathrm{aA}$ & $100,00 \quad \mathrm{aA}$ & $79,00 \quad b B$ \\
\hline 4. Glyphosate+2,4-D $(720+670)$ & $100,00 \quad \mathrm{aA}$ & 100,00 & 100,00 \\
\hline Estimativa de Colby & 100,00 & 100,00 & 99,90 \\
\hline $\begin{array}{l}\text { DMS linha }=8,99 \\
\text { DMS coluna }=9,91 \\
C V \%=7,11\end{array}$ & & & \\
\hline
\end{tabular}




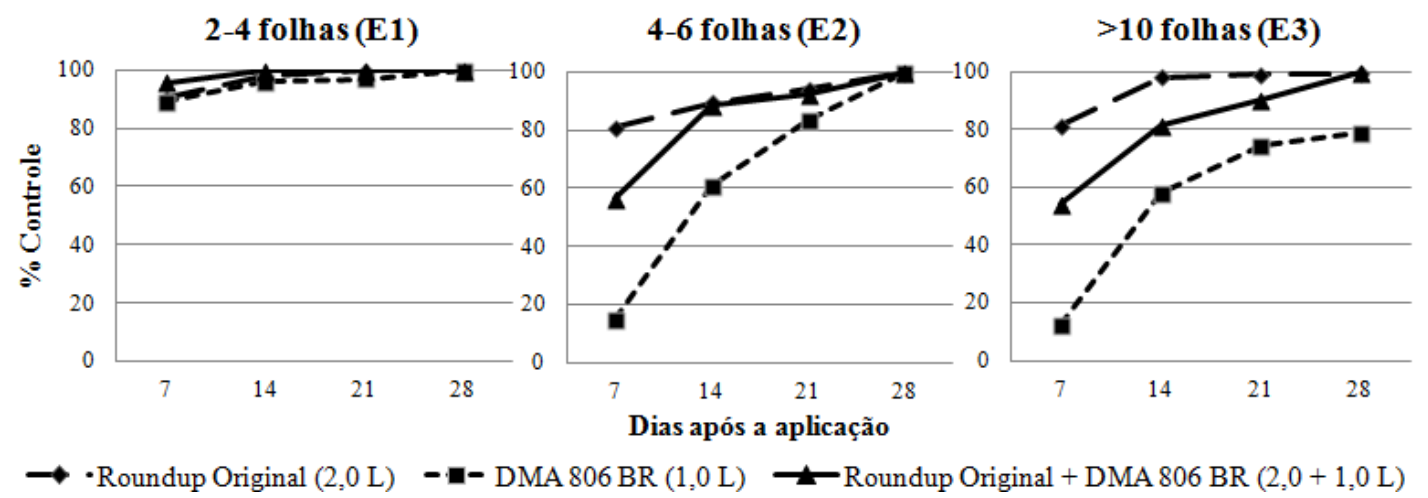

Figura 4. Controle de Spermacoce latifolia após a aplicação de tratamentos herbicidas em três estádios de desenvolvimento ao longo do período de avaliação (Ensaio em casa-de-vegetação). Maringá (PR), 2012.

\section{Corda-de-viola (Ipomoea grandifolia)}

Observou-se aos 28 DAA que somente no estádio E2 a associação entre o glyphosate e o 2,4-D foi superior a aplicação dos herbicidas isoladamente (Tabela 9). Nos demais estádios não houve diferença significativa entre os tratamentos herbicidas. As aplicações isoladas de glyphosate e 2,4-D realizadas quando as plantas encontravam-se no estádio E2 proporcionaram menor controle quando aplicados em estádio E1 e E3.

Nos estádios E1 e E3, a mistura glyphosate+2,4-D apresentou efeio aditivo, visto que o controle foi igual aos valores estimados pelo modelo proposto por Colby (1967). No entanto, para E2 a mistura entre os herbicidas caracterizou-se como sinergística.

No estádio E1, o controle inicial com glyphosate e glyphosate+2,4-D foi semelhante, sendo mais rápido do que o tratamento apenas com 2,4-D. Já no estádio E2 e E3, a velocidade de controle da mistura glyphosate+2,4-D foi superior aos produtos isolados, evidenciando assim o benefício da aplicação dos mesmos em mistura.

Tabela 9. Controle de Ipomoea grandifolia proporcionado pelos tratamentos herbicidas aos 28 DAA (experimento em casa-de-vegetação). Maringá (PR), 2012.

\begin{tabular}{|c|c|c|c|}
\hline \multirow{2}{*}{ Herbicidas e doses (g e.a. ha $^{-1}$ ) } & \multicolumn{3}{|c|}{ Estádio* } \\
\hline & 2-4 folhas (E1) & 4-6 folhas (E2) & $>10$ folhas $(\mathrm{E} 3)$ \\
\hline 1. Testemunha sem herbicida & $0,00 \quad b A$ & $0,00 \quad \mathrm{cA}$ & $0,00 \quad \mathrm{bA}$ \\
\hline 2. Glyphosate (720) & $100,00 \quad \mathrm{aA}$ & 92,20 bB & 96,20 \\
\hline 3. $2,4-\mathrm{D}(670)$ & $100,00 \quad \mathrm{aA}$ & $91,70 \quad b B$ & 100,00 \\
\hline 4. Glyphosate+2,4-D $(720+670)$ & $100,00 \quad \mathrm{aA}$ & 100,00 & 100,00 \\
\hline Estimativa de Colby & 100,00 & 99,34 & 100,00 \\
\hline $\begin{array}{l}\text { DMS linha }=4,66 \\
\text { DMS coluna }=5,14 \\
\text { CV\% }=3,68\end{array}$ & & & \\
\hline
\end{tabular}




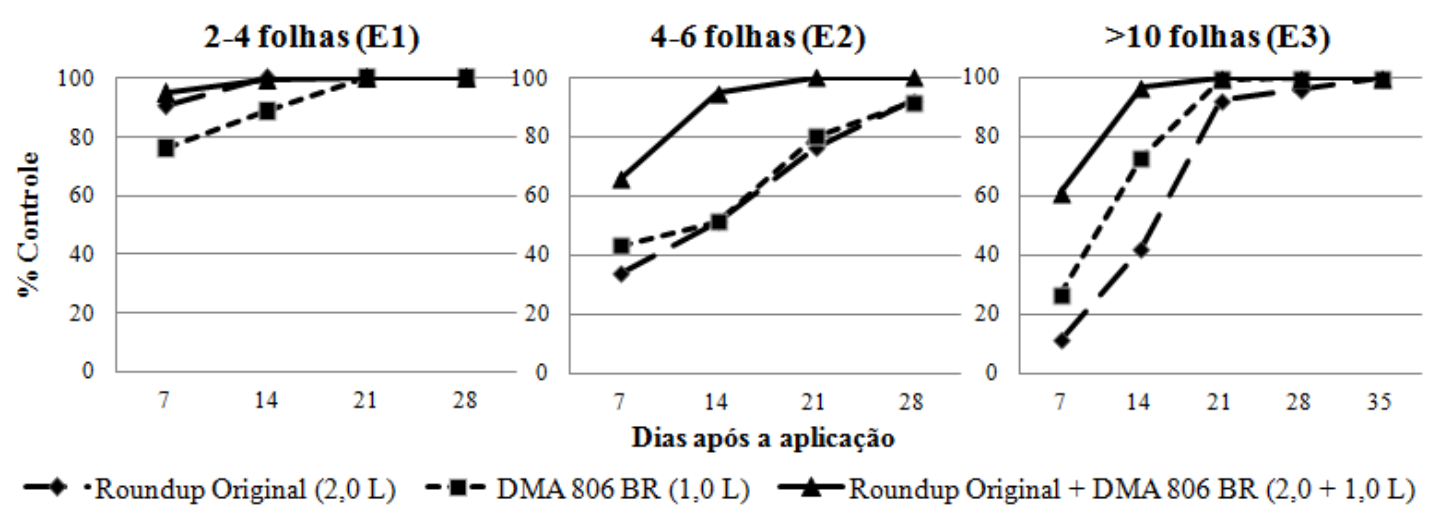

Figura 5. Controle de Ipomoea grandifolia após a aplicação de tratamentos herbicidas em três estádios de desenvolvimento ao longo do período de avaliação (Ensaio em casa-de-vegetação). Maringá (PR), 2012.

\section{Buva (Conyza spp.)}

No experimento realizado em casa-devegetação (Tabela 10), todos os tratamentos controlaram de forma semelhante a buva com menos de $6 \mathrm{~cm}$, com eficiência próxima a $100 \%$. Nesta mesma avaliação, a aplicação de 2,4-D isolado em plantas de Conyza spp. com 6 a $15 \mathrm{~cm}$ proporcionou controle satisfatório (85\%), porém inferior aos demais tratamentos com herbicidas, os quais provocaram a morte total das plantas. Para o estádio E3 $(>15 \mathrm{~cm})$, nenhum tratamento controlou a buva de forma satisfatória, demonstrando a alta tolerância desta aos herbicidas quando aplicados em estádios mais avançados, mesmo em biótipos considerados suscetíveis.

O elevado controle de buva nos estádios E1 e E2 obtido em casa-de-vegetação, ocorreu possivelmente porque as plantas utilizadas neste experimento eram oriundas de uma população suscetível ao glyphosate.

A associação do glyphosate e 2,4-D foi sinergística para o estádio E3 da buva, enquanto que nos demais estádios (E1 e E2) a associação dos herbicidas apresentou efeito aditivo de controle.

O controle das plantas aos 56 DAA, no experimento conduzido em casa-de-vegetação, também não foi satisfatório em nenhum tratamento testado (Tabela 11). As maiores notas de controle foram obtidas pelos tratamentos 5, 6 e 7, variando em torno de
$65 \%$. Os tratamentos 4,3 e 2 obedeceram uma ordem decrescente de eficiência, em que a aplicação de glyphosate isolado novamente proporcionou um nível de controle muito baixo $(6,25 \%)$. De acordo com a equação proposta por Colby (1967), o valor estimado foi de 21,5, sendo assim todos os tratamentos contendo a mistura de glyphosate+2,4-D foram consideradas como sinergísticas para a buva

No ensaio a campo (Tabela 11), o controle exercido pelos tratamentos com herbicidas não foi satisfatório para buva, sendo que o melhor a maior eficiência obtida pela associação de glyphosate+2,4-D (60\%), seguido de 2,4-D (16\%) e glyphosate (6\%). Vale ressaltar que o controle exercido pelo glyphosate foi muito baixo (10\%), evidenciando a resistência dessa planta daninha ao mesmo. Tomando como base o modelo proposto por Colby (1967), a associação de glyphosate+2,4-D foi sinergística para o experimento realizado a campo.

Em todos os estádios de desenvolvimento testados no ensaio a campo, o tratamento com glyphosate+2,4-D proporcionou níveis de controle superiores os produtos aplicados isoladamente (Tabela 12). É importante ressaltar que somente no estádio de até $6 \mathrm{~cm}$ de altura a mistura glyphosate+2,4-D foi eficaz no controle de Conyza spp. (85\%). Nos demais estádios nenhum tratamento obteve controle satisfatório da espécie. Estes 
resultados estão de acordo com Oliveira Neto et al. (2010), os quais relataram excelentes níveis de controle de $C$. bonariensis com glyphosate+2,4-D, realizando em plantas com altura inferior a $6 \mathrm{~cm}$, caracterizando ser o estádio de menor suscetibilidade.

Tomando como base o valor estimado de Colby (1967), a mistura em tanque contendo glyphosate e 2,4-D apresentou efeito sinergístico no controle de buva em todos os estádios de desenvolvimento avaliados.

Com relação à velocidade de controle no experimento em casa-de-vegetação (Figura 6), a associação de glyphosate+2,4-D, independente da dose utilizada proporcionou controle mais rápido em todos os estádios de desenvolvimento avaliados. Vale destacar que em aplicações precoces (estádios E1 e E2) essa diferença na velocidade de controle é menor do que quando aplicados em estádios mais tardios (E3).
No experimento a campo, para todos os estádios de desenvolvimento, a velocidade de controle obedeceu a seguinte ordem decrescente: glyphosate+2,4-D, 2,4-D e glyphosate. Considerando que no campo predominava um biótipo resistente ao glyphosate, nem mesmo plantas pequenas $(<6$ $\mathrm{cm})$ foram eficientemente controladas pelo glyphosate aplicado isoladamente.

Para a buva, o uso da mistura em tanque de glyphosate+2,4-D proporciona um controle mais rápido e mais eficiente em relação aos herbicidas aplicados isoladamente em qualquer estádio de desenvolvimento. Quanto maior o estádio das plantas no momento da aplicação, menor é o controle exercido pelos herbicidas. À campo, para plantas resistentes ao glyphosate nem mesmo a mistura de glyphosate+2,4-D foi eficiente no controle de plantas com mais de 4 folhas.

Tabela 10. Controle de Conyza spp. proporcionado pelos tratamentos herbicidas aos 28 DAA (experimento em casa-de-vegetação). Maringá (PR), 2012.

\begin{tabular}{|c|c|c|c|}
\hline \multirow{2}{*}{ Herbicidas e doses (g e.a. ha $^{-1}$ ) } & \multicolumn{3}{|c|}{ Estádio* } \\
\hline & $<6 \mathrm{~cm}(\mathrm{E} 1)$ & 6-15 cm (E2) & $>15 \mathrm{~cm}(\mathrm{E3})$ \\
\hline 1. Testemunha sem herbicida & $0,00 \quad \mathrm{bA}$ & $0,00 \quad \mathrm{cA}$ & $0,00 \quad \mathrm{cA}$ \\
\hline 2. Glyphosate (720) & $100,00 \quad \mathrm{aA}$ & 100,00 & 23,50 \\
\hline 3. $2,4-\mathrm{D}(670)$ & $99,00 \quad \mathrm{aA}$ & 85,00 & 30,00 \\
\hline 4. Glyphosate+2,4-D $(720+670)$ & $100,00 \quad \mathrm{aA}$ & $100,00 \quad \mathrm{aA}$ & $50,00 \quad \mathrm{aB}$ \\
\hline Estimativa de Colby & 100,00 & 100,00 & 45,00 \\
\hline $\begin{array}{l}\text { DMS linha }=8,83 \\
\text { DMS coluna }=9,73 \\
C V \%=8,93\end{array}$ & & & \\
\hline
\end{tabular}

Tabela 11. Controle final (56 DAA) de Conyza spp. (E3) proporcionado pelos tratamentos herbicidas em casa-de-vegetação. Maringá (PR), 2012.

\begin{tabular}{lrl}
\multicolumn{1}{c}{ Herbicidas e doses $\left(\mathbf{g}\right.$ e.a. $\left.\mathbf{~ h a}^{-\mathbf{1}}\right)$} & \% Controle aos 56 DAA \\
\hline 1. Testemunha sem herbicida & 0,00 & $\mathrm{D}$ \\
2. Glyphosate (720) & 6,25 & $\mathrm{D}$ \\
3. 2,4-D (670) & 16,25 & $\mathrm{C}$ \\
4. Glyphosate+2,4-D (720+670) & 48,75 & $\mathrm{~B}$ \\
5. Glyphosate+2,4-D (720+1005) & 64,25 & $\mathrm{~A}$ \\
6. Glyphosate+2,4-D (900+670) & $65,00 \mathrm{~A}$ \\
7. Glyphosate+2,4-D (900+1005) & $68,50 \mathrm{~A}$ \\
\hline Estimativa de Colby & $\mathbf{2 1 , 5 0}$ & \\
\hline DMS & 9,48 \\
CV\% & 11,12 \\
\hline *Médias seguidas de mesma letra na coluna não diferem entre si pelo teste de Tukey a 5\%.
\end{tabular}




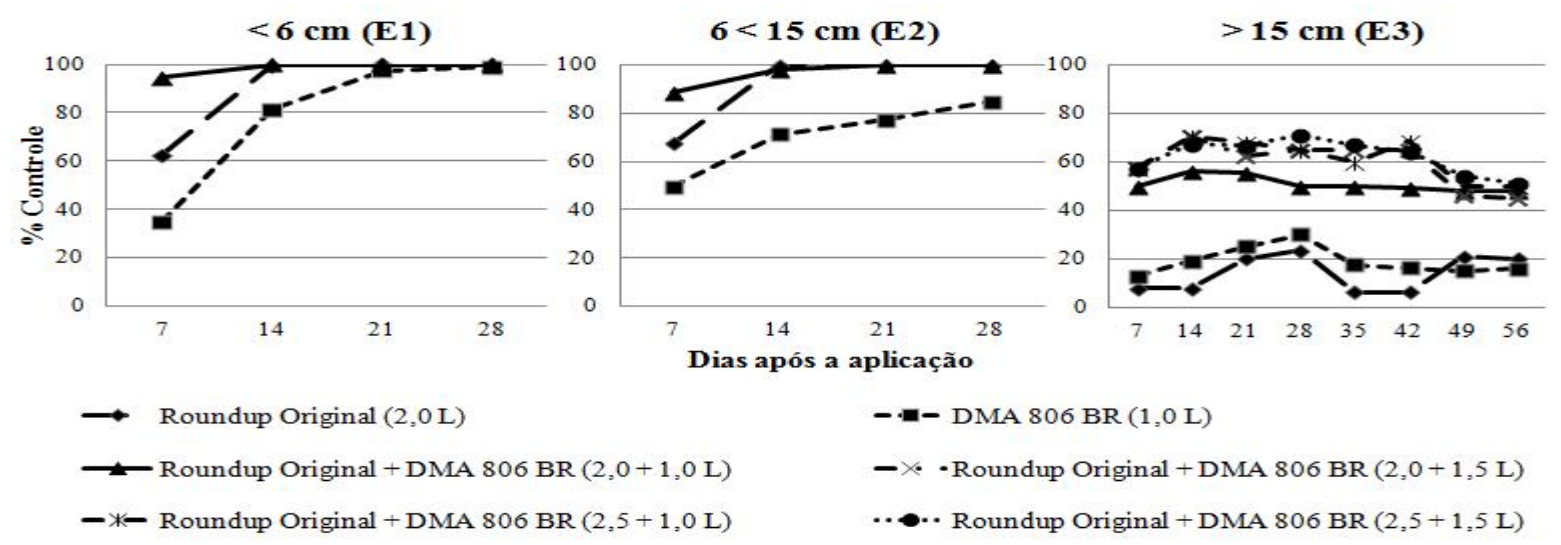

Figura 6. Controle de Conyza spp. após a aplicação de tratamentos herbicidas em três estádios de desenvolvimento ao longo do período de avaliação (Ensaio em casa-de-vegetação). Maringá (PR), 2012.

Tabela 12. Controle de Conyza spp. proporcionado pelos tratamentos herbicidas aos 42 DAA (Ensaio realizado a campo). Maringá (PR), 2012.

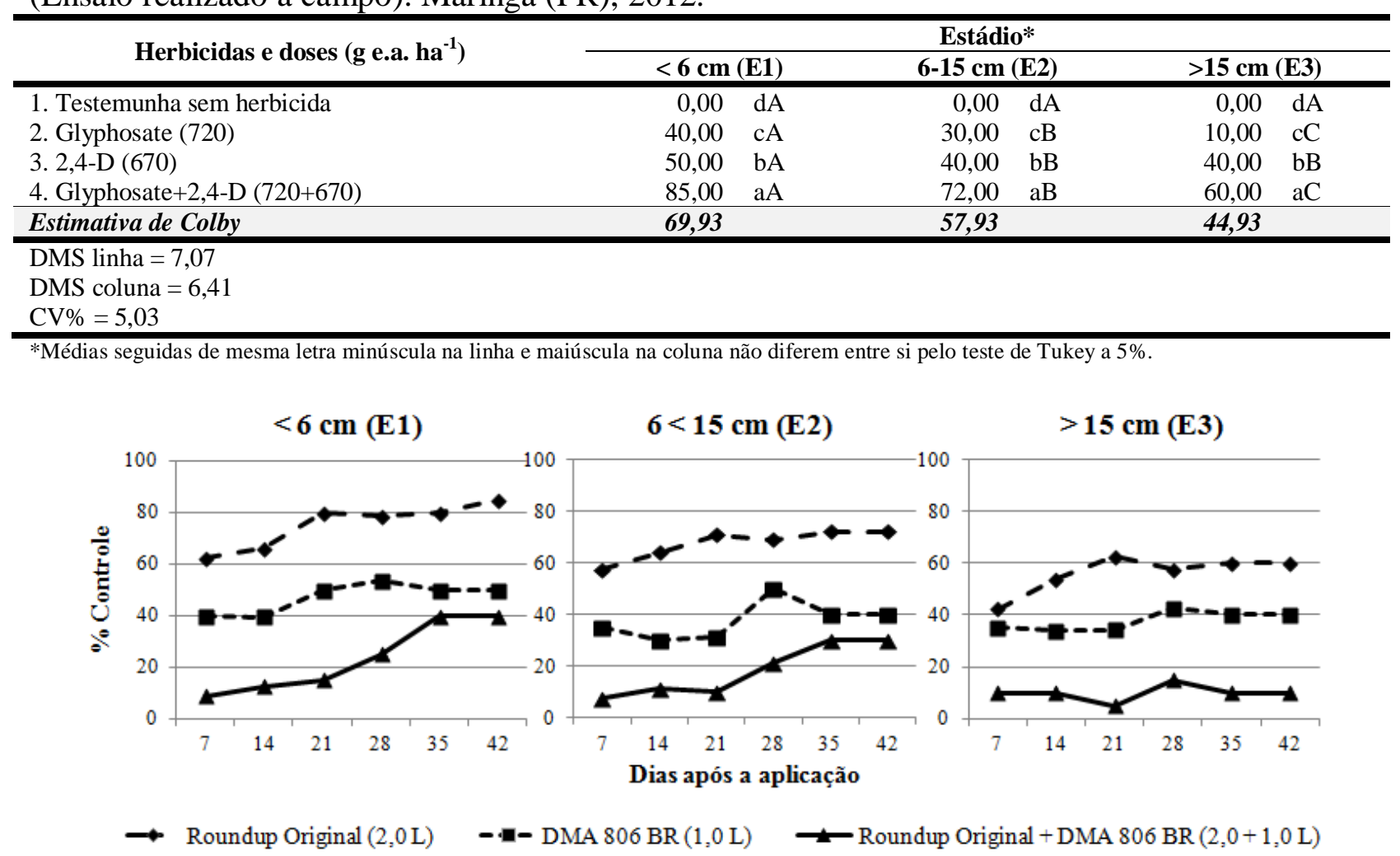

Figura 7. Controle de Conyza spp. após a aplicação de tratamentos herbicidas em três estádios de desenvolvimento ao longo do período de avaliação (Ensaio em campo). Distrito de Pulinópolis, Mandaguaçu (PR), 2012.

\section{Conclusões}

A adição de 2,4-D ao glyphosate é determinante para acelerar e melhorar o controle de plantas daninhas consideradas de difícil controle como as estudadas neste trabalho. 


\section{Referências}

CARVALHO, F.T. et al. Manejo químico das plantas daninhas Euphorbia heterophylla e Bidens pilosa em sistema de plantio direto da cultura de soja. Planta Daninha, v.20, n.1, p.145-150, 2002.

COLBY, S.R. Calculating synergistic and antagonistic responses of herbicides combinations. Weeds, v.15, n.1, p.20-22, 1967.

CORREIA, N.M. et al. Seletividade da soja transgênica tolerante ao glyphosate e eficácia de controle de Commelina benghalensis com herbicidas aplicados isolados e em misturas. Bragantia, v.67, n.3, p.663-671, 2008.

MONQUERO, P.A. et al. Glifosato em mistura com herbicidas alternativos para o manejo de plantas daninhas. Planta Daninha, v. 19, n.3, p. 375-380, 2001.

MONQUERO, P.A.; CURY, J.C.; CHRISTOFFOLETI, P.J. Controle pelo glyphosate e caracterização geral da superfície foliar de Commelina benghalensis, Ipomoea hederifolia, Richardia brasiliensis e Galinsoga parviflora. Planta Daninha, v. 23, n.1, p. 123132, 2005.

OLIVEIRA NETO, A.M. et al. Manejo de Conyza bonariensis com glyphosate $+2,4-\mathrm{D}$ e amônio-glufosinate em função do estádio de desenvolvimento. Revista Brasileira de Herbicidas, v.9, n.3, p.73-80, 2010.

RAMIRES A.C. et al. Glyphosate associado a outros herbicidas no controle de Commelina benghalensis e Spermacoce latifólia. Semina: Ciências Agrárias, v.32, n.3, p.883-896, 2011.

RAMIRES, A.C. et al. Controle de Euphorbia heterophylla e Ipomoea grandifolia com a utilização de glyphosate isolado ou em associação com latifolicidas. Planta Daninha, v.28, n.3, p.621-629, 2010.

RAMOS, H.H.; DURIGAN, J.C. Avaliação da eficácia da mistura pronta de glyphosate $+2,4$ $\mathrm{D}$ no controle da Commelina virginica $\mathrm{L}$. em citros. Planta Daninha, v.14, n.1, p.33-41, 1996.

SANTOS, et al. Buva com resistência múltipla a herbicidas é identificada como Conyza sumatrensis no Paraná. Informe Técnico PGA-Universidade Estadual de Maringá. Maringá, PR, v.1, p.1-3, 2012.

SHARMA, S.D.; SINGH, M. Surfactants increase toxicity of glyphosate and 2,4-D to Brazil pusley. Hortscience, v.36, n.4, p.726728, 2001.

YAMAUTI, M.; BARROSO, A.A.M.; SOUZA, M.C. Controle químico de biótipos de buva (Conyza canadensis e Conyza bonariensis) resistentes ao glyphosate. Revista Ciência Agronômica, v.41, n.3, p. 495-500, 2010. 